\title{
Frédéric Pouillaude D'une graphie qui ne dit rien
}

Les ambiguïtés de la notation chorégraphique

Que la danse soit affaire de présence - présence du corps à lui-même dans l'effectuation du mouvement, présence des danseurs sur la scène dans l'instant fragile de la représentation, ou encore présence réciproque des interprètes et du chorégraphe dans le temps commun de la création -, tout cela fait évidence. Que ce nouage du danser à la présence puisse à lui seul rendre compte du caractère étonnamment marginal et extérieur de la notation en danse, simple accessoire graphique, supplément inutile et étranger, jetant des traces sur le papier, fixant des archives, mais laissant échapper le cœur essentiel de ce qu'il s'agirait d'inscrire, voilà qui demande explication et interprétation.

Bien que disposant depuis la fin du XV siècle de systèmes notationnels plus ou moins élaborés ${ }^{1}$, la danse est demeurée un art fondamentalement oral, un art où les savoirs et les œuvres ne semblent jamais pouvoir se transmettre autrement que de corps à corps, dans la transparence d'un geste nécessairement actuel, et où l'invention et la composition mêmes restent (elles aussi) soumises à l'impératif de présence : inconcevables dans l'espace abstrait et solitaire de la table et de la page, elles ne pourraient advenir que dans le dialogue concret avec le corps présent des interprètes. De fait, la pratique chorégraphique, à la différence de la musique et contrairement à ce que son nom indique, est toujours restée dissociable d'une activité d'écriture et de lecture. Celui que nous appelons aujourd'hui "chorégraphe " n'est précisément pas celui qui écrit, mais celui qui invente, montre et compose. Et le danseur n'est pas non plus celui qui lit (une partition), mais celui qui effectue, reproduit, et invente partiellement lui aussi. Celui qui lit et écrit, c'est un tiers personnage: le notateur ${ }^{2}$. Alors que la tradition musicale occidentale est parvenue à intérioriser son système de notation au point de faire de lire et écrire les quasisynonymes de jouer et composer, la danse aurait quant à elle laissé l'écriture et la lecture au ressort d'une tierce personne, celle-là seule qui ne serait pas analphabète et maîtriserait le système graphique de l'inscription. La scène de l'écriture musicale est, semblable à l'écriture littéraire, solitaire. Celle de la notation chorégraphique serait, étonnamment, triangulaire. Trois instances se trouvent nécessairement réunies: le chorégraphe qui montre et propose, le danseur qui effectue, et le notateur qui inscrit.

Cette extériorité fonctionnelle du notateur, particulièrement évidente aujour- 


\section{Frédéric Pouillaude}

d'hui, n'est sans doute que la conséquence d'une extériorité plus profonde. Toute pratique de danse, toute danse constituée, repose, au même titre que sa notation, sur le découpage et l'identification d'entités discrètes, nommables et répétables; toute danse isole des pas, des figures, des positions; et la constitution d'un tel vocabulaire, même implicite, même transitoire, a pour condition de possibilité l'introduction de discontinuités au sein de l'ensemble des mouvements corporels possibles; pour qu'il y ait danse et vocabulaire, il faut que le continuum infini des mouvements possibles soit démembré, raréfié, de sorte que s'en dégagent quelques êtres identifiables: un "dégagé ", un "développé», un "saut de basque ", une "curve", une "contraction "... La notation graphique n'opère pas autrement, puisque toute institution de signe y fonctionne simultanément comme découpage d'entités, isolation catégorielle et partition du continu. A ce titre, on peut bien dire que toute danse repose sur une archi-écriture, au sens que lui accorde Derrida, une sorte d'articulation première, introduisant des discontinuités dans l'être et fonctionnant comme condition de rappel et d'identification. Le problème est que dans le cas de la danse les deux "écritures", l'archi-écriture du vocabulaire chorégraphique et l'écriture graphique de la notation, n'ont jamais réellement été coextensives. Les partages catégoriels sous-jacents aux signes graphiques sont toujours restés extérieurs, non superposables, aux différentes entités isolées par le vocabulaire chorégraphique. Plus exactement, l'adéquation des deux systèmes n'est jamais advenue que pour de brefs moments.

La Chorégraphie de Raoul-Auger Feuillet ${ }^{3}$ (1700) correspond à l'un de ces moments, sans doute le plus éclatant. Elle inscrit et projette à merveille le vocabulaire de la "belle danse", de cette danse que l'on nomme aujourd'hui de façon sans doute peu appropriée "baroque ». Les découpages catégoriels opérés par le système reproduisaient graphiquement l'archi-écriture du style; ils ne faisaient qu'inscrire sous forme de signes des entités déjà isolées par le vocabulaire. Le système avait alors toutes les chances de devenir réellement immanent aux pratiques qu'il décrivait, puisque ce qui s'épelait sur la page n'était pas différent de ce qui se pensait et s'effectuait en pratique. Et, de fait, la notation Feuillet connut un succès et une diffusion tout à fait remarquables. Editée en 1700, la Chorégraphie est immédiatement réimprimée en 1701, et régulièrement rééditée par la suite. Elle est traduite en anglais dès 1706, et semble faire l'objet d'un consensus à peu près universel durant une soixantaine d'années ${ }^{4}$. Dès 1700 , elle propose, en parallèle avec l'exposé du système, un véritable corpus de danses que chacun peut s'approprier et exécuter pour soi dès lors qu'il sait déchiffrer les signes ${ }^{5}$. Le succès est tel qu'un nouveau corpus paraît en $1706^{6}$. Les «maîtres de ballet » sont alors invités, que ce soit pour des raisons de fixation, de diffusion ou de propriété intellectuelle, à se faire eux-mêmes "chorégraphes ", à noter et inscrire leurs œuvres sous forme de pages écrites que l'on pourra joindre à la partition musicale et au texte du livret. De sorte que, sur cette brève période de temps, il semble bien que la danse ait failli se constituer en une réelle pratique d'écriture et de lecture. L'immanence du système notationnel aux pratiques décrites (l'homogénéité des catégories instituées de part et d'autre) rendait du moins possible une telle constitution. Cependant, cette immanence devait se payer d'une durée de vie nécessairement limitée. 


\section{D'une graphie qui ne dit rien}

L'adhérence du système à un style donné autorisait certes son intégration, mais le condamnait simultanément à devenir obsolète dès que le style aurait lui-même disparu. Ce qui advint, à peu près, autour des années 1750-1760 ${ }^{7}$. Cette dépendance générale de la notation à l'égard d'un style donné expliquerait partiellement l'étonnante abondance des systèmes se succédant les uns aux autres depuis la fin $\mathrm{du} \mathrm{XV}^{\mathrm{e}}$ siècle ${ }^{8}$. Chaque système ne ferait que projeter un vocabulaire particulier et serait de ce fait destiné à périr avec lui, la notation Feuillet étant l'exemple le plus frappant d'un tel phénomène d'adhérence.

Reste la possibilité - ou le fantasme - d'un système réellement universel, inscrivant non pas tel ou tel style, tel ou tel vocabulaire, mais tout mouvement possible en général. Le système serait bien alors capable de prendre en charge tout style de danse, mais au prix d'une nécessaire extériorité. Le système ne peut être système d'inscription de tous les mouvements possibles qu'en instaurant ses propres partages catégoriaux, ses propres entités, indifférentes aux pas, aux figures et aux positions d'un vocabulaire donné. Dès lors, ces catégories doivent faire l'objet d'un apprentissage extérieur et second, disjoint des entités ordinaires sur lesquelles s'appuient les pratiques. Bref, apparaît la figure du notateur. La notation Laban $^{9}$, avec laquelle justement s' instaure cette figure contemporaine du notateur, est l'exemple parfait d'une telle extériorité de l'universel. La cinétographie, autre nom de la notation Laban, parvient à prendre en charge n'importe quel type de mouvement, qu'il soit chorégraphique ou non. Mais elle ne peut le faire qu'en abandonnant toute catégorie locale, tout élément de l'archi-écriture chorégraphique; elle ne peut le faire qu'en instaurant ses catégories propres et en instituant un moment d'arbitraire pur, indifférent aux entités "domestiques" de l'usage. La cinétographie Laban aurait donc connu un échec exactement inverse à celui rencontré par la notation Feuillet : non pas l'adhérence à un style donné se soldant d'une imperméabilité à l'histoire, mais l'inévitable extériorité de toute langue à prétention universelle. La cinétographie Laban constitue aujourd'hui, avec la notation Benesh, l'un des deux principaux systèmes de notation en usage. Mais cet usage opère toujours selon le régime d'extériorité mentionné. Très peu de chorégraphes et de danseurs savent lire ou écrire une partition Laban, et l'enseignement de la notation constitue un domaine tout à fait spécifique et marginal au sein du cursus chorégraphique. Si l'on combine ces deux figures d'échec, l'impossibilité de l'intégration pratique de l'écriture prend alors la forme d'un trilemme: 1 . Pour que l'activité de lecture et d'écriture devienne indissociable des pratiques chorégraphiques, il faut que les découpages catégoriels opérés par la notation soient suffisamment proches des entités mises en ouvres par les pratiques. 2. Cependant, pour que se mette en place une véritable tradition écrite, il faut que le système soit suffisamment large et ouvert, de sorte qu'il supporte la mutation des styles et des genres, et conserve une relative stabilité temporelle. 3. Cette "ouverture» du système - nom affaibli de son universalité - n'est possible que sous condition d'un abandon des catégories locales en usage, ce qui semble contrevenir à la première condition. La cinétographie Laban assumerait les conditions 2 et 3, à l'exclusion de 1 ; la notation Feuillet remplirait exclusivement 1, au détriment de 2 et 3.

Il faut cependant noter que la cinétographie Laban a elle aussi connu son bref 


\section{Frédéric Pouillaude}

moment d'intégration pratique. Les danses de masse et les chœurs de mouvement (Bewegungschöre) de l'Allemagne des années 1920-1930, rassemblant parfois jusqu'à plusieurs milliers de participants, n'auraient sans doute pas pu voir le jour sans une diffusion élargie de la notation et les possibilités d'apprentissage à distance qu'elle offrait ${ }^{10}$. Par ailleurs, le système de décomposition du mouvement sous-jacent à la cinétographie ne se réduit nullement pour Laban à un simple artifice de description. Il s'agit du système réel du mouvement humain, système qui est l'objet même de cette danse dite "moderne " ou "libre " qui s'invente à la même époque et dont le propre est d'œuvrer en deçà de tout code et de tout vocabulaire gestuels établis. La danse "moderne" partage avec la cinétographie un même espace d'universalité où se voient subitement autorisées l'advenue et la prise en compte d'un mouvement réellement quelconque, d'un mouvement non répertorié, inouï. En ce sens, on peut bien dire que les catégories de pensée de la notation étaient destinées à devenir les catégories de la danse elle-même. Seule la cinétographie pouvait être à la hauteur de ce blanc préliminaire que la danse entendait poser comme condition de sa "modernité ". De fait, les concepts de la cinétographie étaient bien à l'origine conçus selon cette règle : tout autant notationnels que chorégraphiques. Le concept de kinesphère - la sphère virtuelle formée par l'ensemble des mouvements des membres autour du corps - est aussi bien une condition de la maîtrise graphique de l'espace (de lui découle la décomposition de l'espace en trente-deux directions élémentaires donnant lieu à trentedeux signes correspondants) qu'un appui analytique pour l'improvisation et la composition. Cependant, ce nouage originaire entre les deux régimes de conceptualité, à supposer qu'il ait jamais eu lieu, s'est progressivement défait. Les concepts labanniens n'ont survécu qu'à travers deux modes de perpétuation parfaitement disjoints : la consignation dans l'implicite du code notationnel, et la série voilée et déformante des transmissions orales. On peut bien retrouver aujourd'hui chez tel chorégraphe ou tel pédagogue la trace de tel concept labannien. Mais jamais selon un lien explicite qui le rattacherait à Laban et donnerait au système notationnel le caractère d'évidence pratique qui lui manque.

Nous aurions donc affaire, avec la notation Feuillet et la cinétographie Laban, à deux formes d'échecs symétriques ${ }^{11}$. Les conséquences de ces échecs ne se limitent pas à l'extériorité fonctionnelle déjà mentionnée. Plus profondément, c'est le statut même de l'œuvre chorégraphique qui est mis en jeu par eux. Ce qui s'annonçait avec l'irruption de la notation, c'était bien la promesse d'une mutation radicale : l'alignement des œuvres chorégraphiques sur le fonctionnement musical et théâtral standard, et l'institution d'un principe d'identification de l'œuvre indépendant de son histoire matérielle de production et de transmission. Bref, c'était la possibilité de faire de l'œuvre chorégraphique un objet idéal fonctionnant comme une classe d'occurrences correctes et autorisant par là des reprises et des recréations extérieures à la série linéaire des transmissions orales. Ou pour le dire encore autrement, en termes goodmaniens cette fois, c'était la possibilité d'un passage de l'autographie à l'allographie qui s'annonçait ainsi. Laban est particulièrement conscient de cet enjeu, et le formule en termes de libération des rapports de production. La notation annonce la fin de l'impératif de présence pesant sur l'exercice 


\section{D'une graphie qui ne dit rien}

de la danse, la fin des liens de dépendance intersubjective qui semblent être à son fondement. Savoir lire et écrire, c'est pour les danseurs se libérer de l'emprise contingente du chorégraphe, et pour le chorégraphe enfin créer indépendamment de la présence non moins contingente des danseurs ${ }^{12}$. Ce motif de l'émancipation se laisse aussi sous-entendre chez Feuillet. Comme l'indique le sous-titre de la Chorégraphie, il s'agit avec la notation de devenir capable d' " apprendre de soi-même toutes sortes de danses ". L'édition simultanée de recueils de partitions, que chacun peut s'approprier comme il l'entend, est là encore révélatrice d'une telle ambition. Cependant, ce motif de l'émancipation par l'écrit doit être nuancé. L'application de la notation aux danses chorales allemandes a marqué à bien des égards la fin des pratiques d'improvisation collective: à l'origine fondées sur l'émergence d'une forme commune née de l'improvisation, les danses chorales vont progressivement devenir la simple exécution de masse d'un texte transcendant et imposé de l'extérieur ${ }^{13}$. De même, la mise en place de la notation Feuillet est indissociable d'une volonté de codification et de préservation du vocabulaire chorégraphique. Pierre Beauchamps, que l'on considère ordinairement comme le véritable inventeur du système exposé dans la Chorégraphie, rapporte, dans le procès qui l'oppose à Feuillet, comment Louis XIV lui avait personnellement confié, une trentaine d'années auparavant, la charge d'inventer une écriture de la danse ${ }^{14}$. Si l'on se fie à ses affirmations, le projet notationnel serait donc à peu près contemporain de la mise en place par Louis XIV en 1661 de l'Académie royale de danse, qui avait précisément pour visée de mettre fin aux débordements stylistiques de la noblesse dansante ${ }^{15}$. Il s'agissait avec l'Académie de soumettre l'exercice de la danse au contrôle de pairs éminents ayant pour charge de codifier le vocabulaire et de le préserver de toute dénaturation. L'inscription graphique du code était conçue comme l'un des instruments possibles de son contrôle, éminemment politique. Rappelons seulement que les Lettres patentes du Roi pour l'établissement de l'Académie Royale de Danse précèdent de quelques mois l'arrestation de Fouquet, et correspondent à ce moment de reprise en main radicale du pouvoir qui caractérise l'accession de Louis XIV à l'exercice personnel de la monarchie. Rappelons que c'est la fête de Vaux-le-Vicomte qui marqua la perte de Fouquet et que cette fête fut aussi un haut moment d'invention chorégraphique avec la mise en place de la première comédie-ballet (Les Fâcheux, de Molière et Lully). L'éclat de la fête et l'invention de nouvelles configurations artistiques ne pouvaient être le fait d'initiatives privées et devaient intégralement revenir à l'Etat. C'est dans ce contexte d'accaparement politique de l'art et de rigidification de son code que doit être réinscrit le projet notationnel. Le motif de l'émancipation par l'écrit est donc à bien des égards discutable. En tout cas, toujours réversible.

Quoi qu'il en soit de ce motif, les promesses de mutation annoncées par la notation - émancipation des rapports de production et institution d'un véritable concept d' œuvre chorégraphique - n'ont jamais été réalisées. L'exercice de la danse est resté inscrit dans l'exigence d'une présence nécessaire. De sorte qu'aujourd'hui les pièces chorégraphiques ne s'inventent nullement sur la page, dans la solitude du bureau, mais à même les corps singuliers d'interprètes choisis, œuvrant d'emblée dans l'espace collectif du studio. Et elles ne se transmettent pas non plus 


\section{Frédéric Pouillaude}

par l'intermédiaire d'un texte qui, fixant une fois pour toutes les critères d'identité de l'œuvre, rendrait superflues les indications des participants originaux, mais bel et bien par l'entremise de passations personnelles, de "reprises de rôles", comme on dit. C'est de cette étonnante dépendance personnelle que découlerait l'ambiguïté de l'œuvre chorégraphique. Ni autographique, ni allographique, ni improvisation éphémère, ni objet idéal, l'œuvre chorégraphique peut se répéter d'un soir ou d'une saison à l'autre, plus ou moins identique à elle-même, sans que cette identité soit jamais fondée en transcendance, dans l'extériorité d'un modèle et d'un texte. Elle possède bien une généralité immanente, qui lui ouvre l'espace de la répétition (pour le dire autrement, elle repose effectivement sur un partage entre propriétés essentielles et contingentes), mais cette généralité et ce partage n'existent pas ailleurs que dans le corps même des interprètes successifs. A la généralité transcendante de la classe subsumant des occurrences, il faudrait donc opposer la généralité immanente de la série s'auto-engendrant perpétuellement, cette dernière généralité étant sans doute en excès sur le partage goodmanien de l'autographie et de l'allographie ${ }^{16}$. Ce statut ambigu de l'œuvre chorégraphique, ni autographique, ni allographique, est la conséquence directe de l'échec notationnel, entendu non comme incapacité descriptive, mais comme impossibilité de l'intégration pratique.

Nous soutenons que cet échec n'est pas un accident historique, mais plus fondamentalement le résultat d'une tension conceptuelle. Et plutôt que d'invoquer abstraitement, comme nous l'avons fait jusqu'à présent, le lien supposé de la danse à la présence, lien qui serait censé frapper de nullité toute tentative d'inscription graphique, nous souhaiterions montrer que la difficulté consiste plus profondément en une tension interne au projet notationnel. L'argument de la présence, comme tel, n'explique rien et n'est guère spécifique. Il s'agit de montrer comment cette exigence de présence se réfléchit au niveau du discours notationnel lui-même et s'y articule comme contradiction. Cette contradiction, nous la repérons dans la référence constante faite à un certain paradigme linguistique, selon lequel la notation chorégraphique devrait être à la danse ce que l'écriture alphabétique est à la langue. Nous soutenons que ce paradigme est essentiellement dialectique. Il articule deux exigences qui, comprises hors langage, risquent de se révéler parfaitement contradictoires : l'exigence "alphabétique " (ou analytique) et l'exigence "sémantique " (ou discursive). L'exigence "alphabétique" demande que les entités chorégraphiques immédiates soient décomposées en éléments simples pouvant faire l'objet d'une combinatoire caractéristique. Pour ce faire, il faut considérer les mouvements produits par la danse comme des entités neutres, non signifiantes, semblables à des phonèmes. A l'inverse, l'exigence "sémantique " requiert que l'inscription graphique ne soit pas seulement une combinatoire de signes servant à noter des entités elles-mêmes non signifiantes; elle exige que la graphie, par delà et à travers la composition élémentaire des phonèmes, soit bien l'inscription d'un discours. A suivre cette seconde exigence, la notation chorégraphique devrait être considérée, au même titre que l'écriture linguistique, comme un "système symbolique d'ordre 2 » : un système notant des signes et non des choses, un système venant 


\section{D'une graphie qui ne dit rien}

doubler un autre système déjà en lui-même signifiant. Il faudrait alors prendre l'analogie au sérieux, et affirmer, au-delà de la seule exigence alphabétique, que la danse est elle aussi, et de plein droit, langage : production de signification et articulation d'un discours. Mais il semble qu'on ne puisse l'affirmer qu'en allant précisément contre les exigences de la décomposition alphabétique, neutralisant les entités et les traitant comme de pures choses. Face à ce nœud problématique, nous soutiendrons que seule l'exigence "alphabétique» est indispensable à la constitution d'une notation chorégraphique, et que cette exigence est au bout du compte parfaitement dissociable de la référence au paradigme linguistique. L'alphabet n'est qu'un exemple parmi d'autres de systèmes symboliques fondés sur la décomposition des entités en éléments simples et leur recomposition par combinatoire caractéristique. Un exemple privilégié, sans doute, et singulièrement complexe puisqu'en lui s'articule aussi l'exigence sémantique ("alphabet », plutôt que de désigner l'une des deux exigences, semble en réalité nommer leur problématique et singulière articulation). Mais, néanmoins, un exemple seulement. Cette thèse portant sur le caractère nécessaire et suffisant de l'exigence analytique, nous la mettrons à l'épreuve de la notation Feuillet.

Et puisque seule l'exigence analytique est nécessaire à la notation, nous soutiendrons corrélativement que la référence au paradigme linguistique dans sa globalité n'intervient le plus souvent que pour faire valoir les droits de la seconde exigence sur la première, que pour disqualifier une écriture décomposant en symboles abstraits le mouvement dansé et laissant échapper les significations essentielles qu'il produit. Faire référence au paradigme linguistique, ce sera la plupart du temps dire que la notation parvient bien à fixer un alphabet du mouvement, mais est condamnée du même coup à tout ignorer du discours qu'il produit.

Cette contradiction issue de la référence à l'écriture alphabétique apparaitra une première fois en extériorité: dans un certain procès que Noverre fait à la notation Feuillet, et reposant tout entier sur l'analogie linguistique. La contradiction se manifestera une seconde fois, en intériorité, au sein du discours labanien. Se référant sans cesse au paradigme de l'écriture linguistique, Laban se voit contraint d'articuler deux systèmes de notation : la cinétographie standard, correspondant à l'exigence analytique et descriptive, et la notation de l'Effort, tentant de prendre en charge l'exigence sémantique et discursive, cherchant à inscrire enfin ce sens du mouvement qui échapperait à la décomposition alphabétique et s'indexerait à l'indépassable présence d'une singularité expressive.

\section{Feuillet : la figure et le caractère}

La notation Feuillet consiste très exactement en l'articulation de deux systèmes symboliques hétérogènes : celui de la figure et celui du caractère. Le titre même de la Chorégraphie (Chorégraphie ou l'art de décrire la dance par caractères, figures et signes démonstratifs) ne doit pas nous induire en erreur. Le troisième terme, 


\section{Frédéric Pouillaude}

"signes", qui d'ailleurs disparaît lors de la reprise du titre à la page 1, n'est ici qu'un nom générique subsumant les deux premiers éléments. Quant au terme "démonstratifs", il fonctionne de façon distributive, s'appliquant aussi bien aux figures qu'aux caractères, selon une forme d'ambiguïté essentielle. Le démonstratif, c'est tout autant ce qui s'exhibe immédiatement dans une représentation iconique - une figure - que ce qui s'inscrit dans une représentation symbolique et une combinatoire - un caractère.

La figure, c'est d'abord la représentation graphique du chemin suivi par les danseurs. Un signifiant, donc. Mais c'est aussi bien ce qui est actualisé sur la scène lorsque les danseurs effectuent le chemin. Un signifié, donc ${ }^{17}$. Pour que les deux termes puissent se recouvrir, il faut que l'espace de la page soit la projection directe de l'espace de la salle. La salle possède un haut, un bas, une droite et une gauche; il en ira de même pour la page, de sorte que la figure graphique n'est rien d'autre que le tracé miniature du chemin. En lisant, il faudra alors faire attention à ce que le livre soit toujours maintenu dans la même orientation : il suffit qu'il bouge d'un simple quart de tour pour que son rapport de projection à la salle soit immédiatement perverti ${ }^{18}$. Car la notation Feuillet est d'abord une représentation planimétrique. L'espace de la page a pour référentiel les coordonnées objectives de la salle, et non la position subjective du corps s'orientant dans l'espace. Il s'agit d'inscrire un chemin objectif - une figure -, et non une séquence d'orientations personnelles (prendre à droite, tourner à gauche, aller tout droit, etc.). La lecture et l'exécution impliqueront donc, outre les artifices nécessaires au maintien du livre dans sa position, une véritable opération de traduction, semblable à celle que suppose l'orientation à partir d'une carte ${ }^{19}$. Il faudra traduire en une suite d'intentions subjectivement orientées ce qui sur la page est seulement représenté par des conséquences spatiales objectives, par un chemin. Et puisque que c'est la salle qui sert de référentiel et non le corps propre, ce qui est figuré sur la page comme une "avancée " pourra très bien être en réalité un pas en arrière ou un pas de côté. Cette dimension planimétrique de la représentation oblige donc à nuancer ce qu'il peut y avoir d'immédiatement "figuratif » dans la figure. Celle-ci est bien l'image et la projection directe du tracé effectué au sol, mais c'est une image qui n'a jamais été vue ni vécue par personne. A supposer que l'on mette de côté la question de l'orientation subjective des danseurs, il faut bien admettre que ce qui est effectivement $v u$ par les spectateurs lors de la représentation, ce n'est pas la figure, ni même le chemin, mais le cheminement entendu comme déploiement temporel d'un tracé. Et la figure, c'est précisément ce qui ressaisit le cheminement sous la forme d'une simultanéité graphique annihilant toute dimension temporelle, sous la forme d'une image. De plus, la figure est la projection d'un point de vue qui n'en est pas un, d'un point de vue qui n'est précisément celui de personne et qui se nomme, depuis Desargues et Leibniz, géométral. Pour voir la figure se dessiner réellement, il aurait fallu se placer "au-dessus " des danseurs, à leur très exacte perpendiculaire, dans ce lieu idéal et infiniment éloigné qu'est le géométral, point de rencontre des droites orthogonales tirées à partir du plan ${ }^{20}$.

Que la figure ne soit jamais vue de personne n'implique pas pour autant qu'il s'agisse d'un pur artifice ou d'une simple abstraction. L'adoption de la représen- 


\section{D'une graphie qui ne dit rien}

tation planimétrique correspond à une tradition chorégraphique réelle. La figure de Feuillet est l'écho lointain d'un certain régime de signification qui fut à son apogée une centaine d'années auparavant. A la charnière du XVI et du XVII siècle, la fonction essentielle d'un ballet est de représenter graphiquement au sol des figures symboliques et géométriques. Le public a alors tout loisir de les déchiffrer, non pas selon le dispositif frontal que nous connaissons aujourd'hui, mais depuis les galeries qui en surplomb entourent la salle. C'est que la danse se pense alors comme écriture immanente et inscription graphique de symboles. L'exemple le plus frappant est sans doute le Ballet de monsieur le duc de Vendosme (1610), resté célèbre pour sa représentation d'un "alphabet des druides ", qui faisait de chaque configuration un véritable hiéroglyphe scénique. Cette fonction géométrique et graphique du ballet est restée plus ou moins en usage durant la première moitié du XVII ${ }^{e}$ siècle, jusqu'à ce que l'élévation progressive de la scène et la disposition frontale du public rendent impossible le déchiffrement ${ }^{21}$. En 1700, la figure a cessé depuis bien longtemps d'être visible sur la scène, et pourtant c'est encore elle qui constitue secrètement la trame de la notation.

Il faut cependant remarquer qu'il n'y a pas toujours une stricte équivalence entre la figure graphique et le chemin effectivement suivi par les danseurs. La figure notée sur la page doit aussi, parallèlement à la représentation immédiate du chemin, manifester l'écoulement temporel : des barres posées à intervalles réguliers sur le tracé viennent marquer la correspondance avec les mesures de la partition musicale, de sorte qu'il faut bien postuler une équivalence parfaite entre déplacement spatial et écoulement temporel. Cette équivalence est nécessairement mise en échec lorsque les danseurs n'évoluent plus dans l'espace et dansent sur place. Alors, la figure graphique cesse temporairement de fonctionner comme chemin et se contente d'exposer une succession temporelle de gestes:

Quand on voudra écrire quelque Dance figurée, on remarquera que tant que la Dance va et ne reste point en une même place, qu’après avoir marqué la présence du corps ou commencement du Chemin de chaque danseur (sic), on dessinera le Chemin sur le papier, semblable à la figure de la Dance, puis on y marquera la Position, et les Pas comme il a été enseigné ci-dessus.

Lorsqu'il arrivera d'écrire plusieurs Pas qui doivent être faits en une même place, comme par exemple au point $A$, on prolongera le Chemin soit sur la même ligne ou d'un côté ou d'autre, selon que l'on trouvera le plus commode, et autant qu'il sera besoin, pour y placer tous les pas qui doivent être faits au point A, lequel Chemin ne sera qu'un Chemin emprunté, afin d'éviter la grande confusion que feraient plusieurs Pas, s'ils étaient écrits sur une même place; je dis donc qu'on prolongera le Chemin comme depuis $\mathrm{A}$ jusqu'à $\mathrm{B}$, et on remarquera que quoique le Chemin ait été prolongé depuis $\mathrm{A}$ jusqu’à $\mathrm{B}$ cependant le Danceur n’aura pas sorti du point $\mathrm{A}$, ce qui se connaîtra facilement par les Pas qui sont depuis $\mathrm{A}$ jusqu’à $\mathrm{B}$, lesquels ne peuvent être faits qu'en une même place ${ }^{22}$.

Sur ce chemin et cette figure globale, il s'agit maintenant d'inscrire des pas. Ce sont les pas qui donnent au chemin sa véritable effectivité (qui temporalisent la 


\section{Frédéric Pouillaude}

figure), et le chemin est lui-même ce en vertu de quoi la figure et les pas peuvent s'articuler :

Le chemin sert à deux usages, premièrement il sert pour écrire les Pas et les Positions, et secondement pour faire observer la Figure des Dances.

A première vue, le pas n'est rien d'autre qu'une petite figure, un petit chemin. C'est la projection au sol du tracé effectué par le pied lors d'un transfert de poids. Nous serions donc encore dans un régime de représentation iconique semblable à celui qui caractérisait la figure. C'est en tout cas ce que laisse penser la nature des signes graphiques attribués aux pas.

Le pas se connaîtra par une petite tête noire représentant l'endroit où est le pied avant de marcher, par une ligne sortant de la petite tête noire représentant sa trace, figure et grandeur, et par un petit revers joint à son extrémité. [...] Quoique la quantité des Pas dont on se sert dans la Dance soit presque innombrable, on les réduit néanmoins à cinq, qui ne serviront ici que pour démontrer toutes les différentes figures que la jambe peut faire, qu'on appelle pas droit, pas ouvert, pas rond, pas tortillé, et pas battu ${ }^{23}$.

Ainsi, au pas droit correspondra une ligne droite (verticale ou horizontale, selon que le pas se fait en avant, en arrière, ou de côté) ; au pas ouvert correspondra un demi-cercle; au pas rond, une sorte de ligne tire-bouchonnée; au pas tortillé, une ligne zigzagante, etc. Petites figures placées comme des accidents de la Figure principale, les signes de pas seraient encore à lire comme images. Cependant, du fait qu'ils désignent des catégories ("pas droit ", "pas ouvert ", etc.) et non des tracés singuliers, ils fonctionnent déjà comme caractères, c'est-à-dire comme signes graphiquement et sémantiquement disjoints ${ }^{24}$. La ligne droite peut être plus ou moins droite, la ligne courbe plus ou moins courbe, les signes n'en désigneront pas moins à chaque fois ce qui est subsumé sous la catégorie "pas droit " ou " pas ouvert ".

Néanmoins, malgré cette amorce de caractéristique, il faut bien voir que les signes de pas fonctionnent encore comme des désignations globales. Ils ne font guère mieux qu'abréger des descriptions verbales, comme le faisaient les systèmes antérieurs d'abréviation tels que le Manuscrit des basses-danses de Marguerite d'Autriche, ou le système proposé par André Lorin quelques années avant Feuillet ${ }^{25}$. C'est seulement au niveau des accidents du pas lui-même, au niveau des petits signes additionnels qui viennent le spécifier, que vont réellement apparaître la décomposition analytique et l'espace de la caractéristique. Le signe de pas se contente d'indiquer un transfert de poids. Mais ce transfert peut s'effectuer et s'enchaîner de bien des façons. Il peut s'agir, pour reprendre les désignations globales en usage, d'un jeté, d'un coupé, d'un contretemps, d'un pas de bourré, d'une cabriolle... Ce sont ces entités "naturelles" que la notation va décomposer en éléments simples, pouvant faire l'objet d'une combinatoire caractéristique. Ces éléments et les signes qui leur sont attribués vont faire perdre à la notation ce qu'il pouvait encore y avoir d'iconique dans la représentation du pas. 
Les pas peuvent être accompagnés des Signes suivants, comme Plié, Elevé, Sauté, Cabriollé, Tombé, Glissé, avoir le pied en l'Air, poser la pointe du Pied, poser le Talon, tourné un quart de Tour, tourné un demi Tour, tourné trois quarts de Tour, et tourné le Tour entier ${ }^{26}$.

Le plié sera représenté par un trait incliné posé sur le signe de pas (« un petit tiré penché du côté de la petite tête noire»), l'élevé par un trait droit ("un petit cran tout droit»), le sauté par deux traits droits, la cabriole par trois traits droits... En outre, ces nouveaux signes nous apprennent que la ligne représentant le pas n'est pas elle-même un élément simple et continu. Elle se compose en réalité de trois zones qui correspondent aux trois moments du pas : son début (avant le transfert), son milieu (pendant le transfert) et sa fin (après le transfert) ${ }^{27}$. Le signe global n'aura pas le même sens selon que le plié ou le sauté intervient au début ou la fin de la ligne. Un contretemps et un jeté sont constitués des mêmes signes élémentaires (un pas modifié par un plié et un sauté), mais different quant au moment (c'est-à-dire quant à l'endroit) où les signes doivent intervenir. Un contretemps est un pas où le saut s'effectue avant le transfert, alors que le jeté est sauté à la fin. Par un simple déplacement de signe, on parvient à représenter de façon fort élégante et économique deux pas qui n’ont pas grand-chose à voir dans l'évidence quotidienne de leur effectuation.

C'est que cette recomposition des entités "naturelles " à partir de leurs signes graphiques simples entend réaliser deux choses: produire en un même signe la désignation univoque du pas et l'exhibition analytique de sa structure. Il ne s'agit pas seulement de trouver une abréviation adéquate, mais de mettre réellement au jour, par l'artifice du signe, une vérité d'ordre structurel. Ce recoupement graphique de la désignation et de l'analyse correspond très exactement au projet de la caractéristique. On a souvent fait référence à l'esprit "cartésien " de la notation Feuillet, toute pétrie d'ordre et de méthode ${ }^{28}$. S'il s'agit ici d'invoquer un nom propre, Leibniz nous semble de très loin préférable, ne serait-ce que pour cet étrange alliage du géométral et de la caractéristique que Feuillet nous donne à lire. Et, plus généralement, c'est selon nous l'espace global de la mathesis classique - mathesis qui, précisément depuis Leibniz, entend aussi être mathesis des ordres qualitatifs - qu'il s'agit de convoquer. La notation Feuillet vise à retrouver par l'artifice graphique et analytique du signe la nature réelle des entités chorégraphiques; elle veut mettre au jour, en deçà des regroupements empiriques et des routines pratiques, les réelles affinités structurelles qui organisent et relient les pas entre eux. Ce faisant, elle assume intégralement le projet de l'épistémè classique, tel qu'exposé par Michel Foucault :

Un système arbitraire de signes doit permettre l'analyse des choses dans leurs éléments les plus simples; il doit décomposer jusqu'à l'origine; mais il doit aussi montrer comment sont possibles les combinaisons de ces éléments, et permettre la genèse idéale de la complexité des choses. [...] Il existe une disposition nécessaire et unique qui traverse toute l'épistémè classique : c'est l'appartenance d'un calcul universel et d'une recherche de l'élémentaire dans un système qui est artificiel, et qui, 


\section{Frédéric Pouillaude}

par là-même, peut faire apparaître la nature depuis ses éléments d'origine jusqu'à la simultanéité de toutes les combinaisons possibles. A l'âge classique se servir de signes, [...] c'est tenter de découvrir le langage arbitraire qui autorisera le déploiement de la nature en son espace, les termes derniers de son analyse et les lois de sa composition $^{29}$.

Cette inscription de la notation au sein de l'épistémè classique se voit confirmée par le statut tout particulier que Feuillet confere au tableau. Avant de rendre possible l'écriture de la danse, la notation doit permettre la classification raisonnée des pas. Entre l'exposé du système et le recueil de partitions, prend place une série de Tables qui, au sein des grandes espèces léguées par la tradition chorégraphique, a pour mission de réordonner les pas en fonction de leur vérité graphique, c'est-à-dire analytique. On trouvera ainsi une Table des Temps de Courante, une Table des demi Coupés, une Table des Coupés, une Table des Jetés, une Table des Contretemps, etc. Ces Tables sont toutes organisées selon un principe de complexité croissante. On part d'une entité élémentaire - le jeté simple, le contretemps simple -, et on lui adjoint progressivement, par combinatoire graphique, l'ensemble de variantes possibles. On peut ainsi manifester des affinités que la pratique ignore, ou même inventer des entités qui ne sont pas en usage. Genèse idéale et classification raisonnée s'identifient alors. De sorte que le lien essentiel de la mathesis et de la taxinomie, mis en évidence par Foucault ${ }^{30}$, se voit ici confirmé.

La notation Feuillet répond donc bien à l'exigence analytique mentionnée plus haut. Décomposant les êtres en éléments simples, elle fonctionne comme une caractéristique : c'est un alphabet. Mais elle ne peut le faire qu'en vertu d'une indifférence totale à l'exigence sémantique : c'est un alphabet qui ne note aucun discours. Les entités de la "belle danse " sont traitées comme choses parmi choses, non signifiantes, décomposables et recomposables à volonté. Non seulement l'exigence analytique est dissociable de l'exigence sémantique (Feuillet ne fait pas une seule fois référence au paradigme linguistique qui est au fondement de leur association), mais plus radicalement il semble même que l'analyse ait pour condition de possibilité la suspension du sens, la mise entre parenthèses de tout le réseau de correspondances et de significations qui, à la Renaissance et encore à l'âge classique, faisait de la danse une "rhétorique muette ", une représentation allégorique de figures, voire une inscription scénique de l'harmonie des sphères ${ }^{31}$. Pour que se mette en place la notation Feuillet, il aura fallu en quelque sorte neutraliser les pas et les gestes, les soustraire à toute prétention signifiante, et les traiter comme des choses. Répertoire de pas, et non dépositaire d'un sens, description de figures, et non écriture d'une langue, le système s'entend alors comme alphabet sans discours. Une soixantaine d'années plus tard, Noverre ne manquera pas de pointer le paradoxe, faisant alors du paradigme linguistique le levier même de sa critique. 


\section{D'une graphie qui ne dit rien}

\section{Noverre : la lettre et la parole}

C'est bien un nouveau régime de signification que Noverre entend fonder dans ses Lettres sur la danse et les ballets (1760), un régime qui indexerait la danse non pas à la représentation d'une figure ou d'un signe, mais à l'expression directe des passions. Ce nouveau régime, il le nomme, en reprenant l'expression de Cahu$\mathrm{sac}^{32}$, danse en action.

L'action en matière de danse est l'art de faire passer par l'expression vraie de nos mouvements, de nos gestes et de la physionomie, nos sentiments et nos passions dans l'âme des spectateurs ${ }^{33}$.

Cette danse en action ne s'entend que sur fond de dualisme radical, le dualisme du mécanique et de l'expressif, du muet et du parlant, du physique et du moral :

La danse proprement dite se borne uniquement au mécanisme des pas et des mouvements des bras; dès lors, elle ne peut être regardée que comme une profession, dont le succès se borne à l'adresse, à l'agilité, à la vigueur et l'élévation plus ou moins grande des cabrioles. Mais lorsque l'action pantomime se réunit à tous ces mouvements machinaux, la danse acquiert un caractère de vie qui la rend intéressante; elle parle, elle exprime, elle peint les passions et mérite alors d'être rangée dans la classe des arts imitateurs ${ }^{34}$.

Je diviserai la danse en deux classes; la première, danse mécanique ou d'exécution; la seconde, danse pantomime ou en action.

La première ne parle qu'aux yeux, et les charme par la symétrie de ses mouvements, par le brillant des pas et la variété des temps; par l'élévation du corps, l'aplomb, la fermeté, l'élégance des attitudes, la noblesse des position, et la bonne grâce de la personne. Ceci n'offre que la partie matérielle.

La seconde, que l'on nomme danse en action, est, si j'ose m'exprimer ainsi, l'âme de la première; elle lui donne la vie et l'expression, et en séduisant l'œil elle captive le cœur, et l'entraîne aux plus vives émotions; voilà qui constitue l'art ${ }^{35}$.

Il s'agit donc de faire parler la danse. Car, auparavant, elle était muette ${ }^{36}$. Toute préoccupée de l'enchaînement des pas, du respect des positions et de la complexité des figures - choses que nous enseignait Feuillet -, elle restait prise dans une pure mécanique, flattant certes le regard, mais n'exprimant rien. Le sens ne pouvait lui être adjoint que de l'extérieur, par l'entremise du livret ou des dialogues:

Ce n'est pas d'aujourd'hui que l'on donne le titre de ballet à des danses figurées que l'on ne devrait appeler que du nom de divertissement; on prodigua jadis ce titre à toutes les fêtes éclatantes qui se donnèrent dans les différentes cours de l'Europe. 


\section{Frédéric Pouillaude}

L'examen que j'ai fait de toutes ces fêtes me persuade que l'on a eu tort de le leur accorder. Je n'y ai jamais vu la danse en action; les grands récits étaient mis en usage au défaut de l'expression des danseurs, pour avertir le spectateur de ce qu'on allait représenter; preuve très claire et très convaincante de leur ignorance, ainsi que du silence et de l'inefficacité de leurs mouvements. [...] Sous le régime de Louis XIV, les récits, les dialogues et les monologues ne servaient-ils pas d'interprètes à la danse? Elle ne faisait que bégayer ${ }^{37}$.

Cette caractérisation du ballet de cour est à bien des égards réductrice. Le ballet de cour de Louis XIV prétend tout autant à l'imitation que la danse en action de Noverre. Ménestrier ne cesse de le répéter : la danse est un art d'imitation ${ }^{38}$. Simplement, c'est le concept même d'imitation qui, de Ménestrier à Noverre, s'est trouvé bouleversé. Si le ballet de cour n'imite qu'en prenant appui sur les éléments annexes de la représentation, sur le costume, le masque, les décors, le texte..., c'est précisément parce qu'il ne cherche pas à exhiber des individus ou des affects, mais à produire des figures symboliques, des signes instantanés, que le regard des spectateurs se fera un plaisir de déchiffrer et de reconnaître. Ainsi on peut sans aucun risque d'invraisemblance danser le Vent, le Soleil, la Colère, la Paix, ou le mouvement des Astres. Chose parfaitement inconcevable pour Noverre. Il ne s'agit pas ici d'une parole expressive, d'une émotion montrée dans son déploiement temporel, mais d'une image offerte comme un instantané à déchiffrer, ce que met fort bien en évidence Cahusac dans les propres critiques qu'il formule à l'égard du ballet de cour :

Dans le grand ballet, il y a beaucoup de mouvements, et point d'action. La danse peut bien y peindre par des habits, par des pas, par des attitudes, des caractères nationaux, quelques personnages de la fable ou de l'histoire; mais sa peinture ressemble alors à la peinture ordinaire qui ne peut rendre qu'un seul moment, et le théâtre est fait par sa nature pour représenter une suite de moments, de l'ensemble desquels il résulte un tableau vivant et successif qui ressemble à la vie humaine. [...]

Les Furies dans une Entrée particulière, par exemple, pouvaient sans doute, par des pas rapides, par des sauts précipités, par des tourbillons violents, peindre la rage qui les agite, mais ce n'était qu'un trait général, un coup de pinceau épisodique. Il en résultait qu'on avait vu les Furies et rien de plus ${ }^{39}$.

Le ballet de cour, donc, soit se contente de la pure virtuosité insignifiante des pas, soit élabore des signes hétérogènes où la danse n'est qu'un élément parmi d'autres. En aucun cas, la danse n'y parle. Faire parler la danse, pour Noverre, c'est parvenir à métamorphoser la mécanique élémentaire, réussir à la combiner de telle sorte que s'en dégage miraculeusement un discours. Et c'est là qu'intervient de nouveau la métaphore de l'écriture, l'image de l'alphabet et de la langue :

[Face au danseur mécanique] j'applaudis alors à l'adresse, j'admire l'hommemachine, je rends justice à sa force, à son agilité; mais il ne me fait éprouver 


\section{D'une graphie qui ne dit rien}

aucune agitation; il ne m'attendrit pas et ne me cause pas plus de sensation que l'arrangement des mots suivants : fait... pas... le... la... honte... non... crime... et... l'échafaud. Cependant, ces mots arrangés par le grand homme composent ce beau vers du Comte d'Essex :

Le crime fait la honte et non pas l'échafaud.

Il faut conclure de cette comparaison que la danse renferme en elle tout ce qui est nécessaire au beau langage et qu'il ne suffit pas d'en connaître l'alphabet. Qu'un homme de génie arrange les lettres, forme et lie les mots, elle cessera d'être muette, elle parlera avec autant de force que d'énergie, et les ballets alors partageront avec les meilleures pièces de théâtre la gloire de toucher, d'attendrir, de faire couler des larmes $[\ldots]^{40}$.

Les pas sont comme des lettres insignifiantes, ou des mots atomisés, qu'il s'agit de combiner adéquatement selon une formule qui permettrait enfin de les faire parler. Cette métaphore de la lettre ou de l'écriture se retrouve chez Cahusac, selon le même usage, mais sous des formes diverses. Tantôt le mauvais danseur est comparé au sage chinois, dont tout le savoir consiste en la connaissance d'idéogrammes isolés, que jamais il n'articule en un discours. Ce sage aurait bien tous les éléments du savoir, mais sous la forme du non-savoir absolu, sous la forme de l'éparpillement rhapsodique et de la représentation atomique. Il en irait à peu près de même du danseur qui n'est que mécanique : il connaîtrait sur le bout des doigts l'alphabet du mouvement, mais, précisément parce qu'il ignore la langue, en viendrait à traiter les lettres comme des idéogrammes, absurdement. Ailleurs, c'est au Maître Ecrivain que ce danseur est comparé, à celui qui, maîtrisant exemplairement la graphie des lettres, n'est justement pas celui qui maîtrise le discours ${ }^{41}$.

Cette métaphore de l'écriture est cependant trompeuse; elle laisse croire que le sens du mouvement pourrait résulter d'une simple combinatoire de l'insignifiant, à peu près comme le font les lettres lorsqu'elles se combinent en mots. Noverre soutient en réalité exactement le contraire : la signification chorégraphique est radicalement hétérogène à son alphabet, et ne peut se manifester quà un autre niveau que celui des pas et des figures. L'écriture alphabétique ne joue pas ici le rôle d'un paradigme à proprement parler, mais seulement celui d'une métaphore critique. Cette métaphore permet d'affirmer qu'une danse purement mécanique est aussi absurde que la maîtrise d'un alphabet qui ne s'accompagnerait pas d'une compétence linguistique. Mais, en réalité, comme rien dans cet alphabet et les combinaisons auxquelles il donne lieu ne permet l'apparition d'un sens, une telle disjonction est parfaitement normale. C'est ailleurs que le sens doit apparaître, dans un "ailleurs " proprement topologique. Alors que l'alphabet du mouvement se concentre sur les jambes, sur l'enchaînement des pas et des figures, le sens, quant à lui, ce par quoi la danse devient parole et expression, a pour site les bras et le visage. Noverre ne cesse de marteler qu'il faut accorder plus d'attention aux bras et moins de valeur aux jambes :

Pour hâter les progrès de notre art et le rapprocher de la vérité, il faut faire un sacrifice de tous les pas trop compliqués; ce que l'on perdra du côté des jambes se 


\section{Frédéric Pouillaude}

retrouvera du côté des bras. [...] Je demande plus de variété et d'expression dans les bras; je voudrais les voir parler avec plus d'énergie; ils peignent le sentiment et la volupté, mais ce n'est pas assez, il faut encore qu'il peigne la fureur, la jalousie, le dépit, l'inconstance, la douleur, la vengeance, l'ironie, toutes les passions innées enfin dans l'homme et que, d'accord avec les yeux, la physionomie et les pas, ils me fassent entendre le cri de la nature ${ }^{42}$.

Quant au visage, lieu éminent de l'expression des passions, Noverre se félicite de lui avoir rendu sa transparence en proscrivant l'usage des masques :

J'ai eu le courage de proscrire les masques du théâtre et ils n'osent plus se montrer qu'aux bals; $j$ 'ai toujours regardé ces masques de bois ou de cire comme une enveloppe épaisse et grossière qui étouffe les affections de l'âme et ne lui permet pas de manifester au dehors les impressions qu'elle ressent ${ }^{43}$.

Cette apparition du sens, cet "ailleurs" du pas que sont les mouvements des bras et la mimique du visage, s'ordonne à la mise en place d'un nouveau concept : celui de geste, et d'une nouvelle logique : celle de l'expression ${ }^{44}$. Le geste expressif ne peut faire l'objet d'aucune codification préalable. Il ne s'agit pas d'une entité chorégraphique pré-donnée que l'on pourrait se contenter d'exécuter, mais bien plutôt de l'exhibition directe d'une intériorité singulière et passionnée, de son exposition sous forme de mouvements infimes et infiniment variés :

Le port des bras devant être aussi varié que les différentes passions que la danse peut exprimer, les règles reçues deviennent presque inutiles; il faudrait les enfreindre et s'en écarter à chaque instant ou s'opposer en les suivant exactement aux mouvements de l'âme, qui ne peuvent se limiter par un nombre déterminé de gestes. [...] Le geste puise son principe dans la passion qu'il doit rendre; c'est un trait qui part de l'âme, il doit faire prompt effet et toucher au but lorsqu'il est lancé par le sentiment. Instruit des principes fondamentaux de notre art, suivons les mouvements de notre âme, elle ne peut nous trahir lorsqu'elle sent vivement; et si, dans ces instants, elle entraîne le bras à tel ou tel geste, il est toujours aussi juste que correctement dessiné et son effet est sûr. Les passions sont les ressorts qui font jouer la machine : quels que soient les mouvements qui en résultent, ils ne peuvent manquer d'être vrais. Il faut conclure d'après cela que les préceptes stériles de l'école doivent disparaître dans la danse en action pour faire place à la nature ${ }^{45}$.

La logique du geste nous exporte nécessairement hors de l'alphabet. Elle nous confronte à la singularité du sujet dansant et à la vérité tautologique de l'expression. Par quoi s'évanouit toute l'extériorité d'un vocabulaire. Elle nous affronte à la continuité des signes; infiniment variés, les symptômes expressifs ne peuvent faire l'objet d'aucune catégorisation discontinue. Par quoi disparât aussi l'idée de caractère ou d'entité. Elle implique la simultanéité du sens et de son énonciation; pas d'expression, hors d'une passion intérieurement et présentement vécue. Par quoi enfin fait retour le nouage du danser à la présence. 


\section{D'une graphie qui ne dit rien}

Nous sommes désormais armés pour entendre le procès que Noverre fait à Feuillet ${ }^{46}$. Il tient en une phrase. La Chorégraphie "épelle la danse».

La lettre s'ouvre sur un résumé des principes, qui d'un même mouvement condamne et l'alphabet et le géométral :

Les chemins ou la figure de ces danses étaient tracées; les pas étaient ensuite indiqués sur ces chemins par des traits et des signes démonstratifs et de convention; la cadence ou la mesure étaient marqués par de petites barres posées transversalement qui divisaient les pas et fixaient les temps; l'air sur lequel ces pas étaient composés, se notait au-dessus de la page, de sorte que huit mesures de Chorégraphie équivalaient à huit mesures de musique; moyennant cet arrangement on parvenait à épeler la danse, pourvu que l'on eût la précaution de ne jamais changer la position du livre, et de le tenir toujours dans le même sens ${ }^{47}$.

La notation ne retient du mouvement dansé que son élément alphabétique, cela seul qui peut entrer dans l'explicite du code et la discontinuité du vocabulaire: " cet art n'indique exactement que l'action des pieds 48 ". Les mouvements subtils des bras, les effacements du buste, les oppositions de la tête, les expressions du visage, qui font l'essentiel de la signification chorégraphique, ne peuvent y prendre place. Le grain de la notation n'est pas assez fin pour prendre en charge le lieu même de l'expressivité. Il ne s'agit pas là d'une difficulté contingente, à laquelle on pourrait remédier en affinant et complexifiant le système, mais bien d'une impossibilité d'essence, reposant sur le dualisme du mécanique et de l'expressif. La notation assume le mécanique, et l'épelle. Même si à ce niveau elle semble déjà être dépassée ("aujourd'hui les pas sont compliqués; il est donc très difficile de les mettre par écrit et encore plus difficile de les déchiffrer ${ }^{49} »$ ), elle peut toujours rajouter des signes complémentaires, inventer des artifices graphiques et complexifier à l'envi. Là où elle bute irrémédiablement, c'est sur la signification comme telle, sur cette expression qui échappe à tout code, à toute catégorie, et ne peut se concevoir hors d'une actualisation pleine et entière. L'essentiel de la danse est dans la présence en acte d'une passion qui s'exporte, et cela ne se note guère.

C'est pourquoi les partitions Feuillet fonctionnent en réalité comme les vestiges et les traces d'un événement dérobé, et nullement comme des corpus consignant l'identité des œuvres :

Entassez, tant qu'il vous plaira, ces faibles monuments de la gloire de nos danseurs célèbres; je n'y vois et l'on n'y verra que le premier crayon, ou la première pensée de leurs talents; [...] j'apercevrais seulement des vestiges et des traces d'une action dans les pieds que n'accompagneront ni les attitudes du corps, ni les positions des bras, ni l'expression des têtes; en un mot, vous ne m'offrirez que l'ombre imparfaite du mérite supérieur, et qu'une copie froide et muette d'originaux inimitables ${ }^{50}$.

C'est bien là tout l'arsenal platonicien du Phèdre qui est repris : la métaphore de l'ombre et de la lumière, de la copie et de l'original, de ce qui parle et de ce qui 


\section{Frédéric Pouillaude}

reste muet. Cet impératif de la parole vive, de la nécessaire présence du sens à luimême, il faut aussi l'appliquer à la création :

C'est une erreur que de penser qu'un bon maître de ballet puisse tracer et composer son ouvrage au coin du feu. Ceux qui travaillent ainsi ne parviendront jamais qu'à des combinaisons misérables. Ce n'est pas la plume à la main qu'on fait marcher les figurants. [...] Le théâtre est le Parnasse des compositeurs ingénieux; c'est là que sans chercher, ils rencontrent une multitude de choses neuves; tout s'y lie, tout est plein d'âme, tout y est dessiné avec des traits de feu. [...] La conduite et la marche d'un grand ballet bien dessiné exige, Monsieur, des connaissances, de l'esprit, du génie, de la finesse, un tact sûr, une prévoyance sage et un coup d'œil infaillible, et toutes ces qualités ne s'acquièrent pas en déchiffrant et en écrivant la danse chorégraphiquement; le moment seul détermine la composition; l'habilité consiste à le saisir et à en profiter heureusement ${ }^{51}$.

Composer, cela ne peut avoir de sens que dans le théâtre lui-même et la présence simultanée des participants. Car composer, c'est avant tout savoir s'emparer d'un présent contingent, d'un accident heureux, d'un moment imprévu, d'un kairos. Notons que cette logique du kairos chorégraphique gouverne encore profondément notre modernité, y compris et peut-être surtout ses franges les plus «avancées » : composition instantanée, adaptation au site, attente de l'accident..., bref, tout ce qui sous le nom de performance rend volontairement la danse indissociable de son inscription événementielle.

Cependant, Noverre ne dévalue pas absolument l'inscription graphique. Si l'on renonce à la valeur de corpus des partitions et qu'on les considère comme de simples traces ou archives, des améliorations peuvent alors être envisagées. Critiquant l'article "Chorégraphie» rédigé par Cahusac pour l'Encyclopédie, Noverre en propose quelques-unes. Cahusac se contente d'un exposé neutre du système Beauchamps-Feuillet et adjoint simplement quelques planches tirées de la Chorégraphie. Noverre envisage un projet d'une tout autre ampleur, reposant en réalité sur un retour pur et simple à la représentation picturale et la description verbale, et impliquant par là-même une dévaluation radicale des mérites de la représentation planimétrique et géométrale. Le plan géométral, outre qu'il contraint le lecteur à tourner autour du livre, n'offre qu'une vue d'oiseau de la figure principale. Cette vue d'oiseau laisse échapper le réel de la représentation, ce qui est effectivement $v u$ depuis le parterre, toujours frontalement, selon le plan d'élévation. Lequel, en réalité, n'est même pas un plan, mais bien une vue perspective. C'est pourquoi Noverre propose d'adjoindre à ce qui est noté sur le plan géométral une représentation purement picturale de la scène, une gravure qui donnerait à voir l'image réelle des figures, des situations, des personnages, des costumes et des expressions. Cette représentation picturale, Noverre la nomme, assez improprement, mais par souci de parallélisme avec le plan géométral, plan d'élévation. Et, à ce plan d'élévation, qui n'est en réalité qu'une vue perspective, il s'agira encore d'adjoindre des descriptions verbales. Bref, on multipliera les angles d'attaque, on fera flèche de tout bois, notation, dessin, discours, non pas pour fixer l'identité d'une œuvre 


\section{D'une graphie qui ne dit rien}

qui pourrait se transmettre selon les procédures d'absence autorisées par le texte, mais seulement pour laisser aux générations futures l'archive ou la trace d'une présence indépassable:

La chorégraphie deviendrait alors intéressante. Plan géométral, plan d'élévation, description fidèle de ces plans, tout se présenterait à l'œil avec les traits du goût et du génie; tout instruirait, les attitudes du corps, l'expression des têtes, les contours des bras, la position des jambes, l'élégance du vêtement, la vérité du costume; en un mot, un tel ouvrage soutenu du crayon et du burin de ces deux illustres artistes serait une source où l'on pourrait puiser, et je le regarderais comme les archives de tout ce que notre art peut offrir de lumineux, d'intéressant et de beau ${ }^{52}$.

Du corpus à l'archive, tel est le mouvement que Noverre fait subir à la notation. Et c'est là la conséquence directe du jeu dialectique de l'écriture. Dès lors que la notation est conçue comme l'écriture d'une langue, elle se trouve nécessairement jugée à l'aune d'une prétention qu'elle ne peut remplir. Graphie qui ne dit rien, elle ne peut qu'inscrire le squelette d'un sens extérieur, et devenir la trace d'une présence nécessairement disparue.

\section{Laban : le mouvement et l'Effort}

La position de Laban se déduit d'un fait simple : une égale admiration à l'égard de Feuillet et de Noverre ${ }^{53}$. Cette double filiation suppose très exactement que le paradigme linguistique fasse retour en intériorité: non comme levier d'une critique externe, mais comme métaphore d'une résolution fantasmée. L'écriture linguistique, c'est ce qui s'annonce comme la miraculeuse concordance de l'analyse et du sens, de l'alphabet et de la parole, concordance qui toujours manque à la notation :

Une notation chorégraphique neuve et universellement acceptable, semblable à l'alphabet phonétique, devait être construite. Des images ou des diagrammes représentant la forme extérieure du mouvement et des positions corporelles ne pouvaient réaliser cette fin dernière qu'est la notation du contenu spirituel des danses ${ }^{54}$.

Puisqu'elle est une composition de mouvements, la danse peut être comparée au langage parlé. De même que les mots sont formés de lettres, les mouvements sont formés d'éléments. De même que les phrases sont constituées de mots, celles de la danse le sont de mouvements ${ }^{55}$.

De même que les lettres de l'alphabet composent des mots qui s'organisent en phrases, les éléments simples de mouvement composent des segments plus élaborés et finalement des phrases de danse ${ }^{56}$. 


\section{Frédéric Pouillaude}

Si la danse est constituée comme une langue, faite d'éléments simples devenant signifiants par les pures vertus de la combinatoire, la notation peut alors assumer d'être pur alphabet. Elle sera du même coup, automatiquement, transcription d'un discours. C'est bien cet idéal qui semble gouverner la cinétographie, laquelle n'est rien d'autre qu'un système de décomposition du mouvement ${ }^{57}$. Conformément à la kinesphère, l'espace subjectif est divisé en trente-deux signes directionnels simples, et conjointement, le corps est décomposé selon ses principales zones motrices et articulaires, correspondant chacune aux différentes colonnes de la partition. Grâce à cette double analytique, on parvient à représenter de façon purement symbolique n'importe quelle posture corporelle, le mouvement s'entendant alors comme passage implicite d'une posture à une autre. Les signes d'appui permettent quant à eux de représenter les transferts de poids, et donc les déplacements dans l'espace.

La cinétographie Laban rompt avec la notation Feuillet en ce qu'elle abandonne la représentation planimétrique. La page n'est plus la projection de l'espace, mais seulement celle du temps. La partition, qui se lit de bas en haut, déploie une séquence de gestes et de déplacements, dont le caractère spatial n'est assumé que sous forme symbolique et non iconique. En outre, cette spatialité n'est pas celle d'un référentiel extérieur, mais celle qui s'oriente à partir du corps propre. C'est une séquence d'instructions motrices subjectivement référées qui est notée, et non la figuration immédiate d'un chemin. Les positions dans l'espace objectif (c'est-à-dire rapportées à un référentiel extérieur) ne sont lisibles qu’à titre de conséquences implicites. Alors que la notation Feuillet articulait deux systèmes hétérogènes, la figure et le caractère, la représentation iconique du chemin et la représentation symbolique des pas, la cinétographies'appuie quant à elle sur la seule puissance analytique et combinatoire de symboles abstraits. Nous aurions donc bien affaire à un parfait alphabet, et d'autant plus parfait qu'il semble pouvoir prendre en charge n'importe quel type de mouvement.

Une difficulté, cependant. Toujours la même. C'est en réalité un alphabet qui ne dit rien. Il note la face objective du mouvement, les tracés, les postures, les temps; il inscrit des paramètres quantitatifs : vitesse, direction, amplitude..., mais laisse nécessairement de côté ce qu'on nommera ici grossièrement le qualitatif, l'accompagnement subjectif du mouvement. Cet accompagnement, évident pour quiconque fait la différence entre la chute d'une pierre et l'expression d'un vivant, Laban le nomme justement expression ou couleur, mood: tonalité affective, et, simultanément, visibilité d'un état. Et c'est ce mood qui doit être mis au fondement de la signification chorégraphique : "La signification des phrases de mouvement peut être comprise comme l'expression de "couleurs" [moods] d'actions définies" ("The sense of the phrases of movement can be understood as the expression of definite action moods" $)^{58}$.

Ce mood, cette face qualitative et subjective du mouvement qui est le principe même de sa "coloration", n’a que partiellement à voir avec l'extériorité des postures et des gestes:

Les couleurs [moods] ou expressions des mouvements ont une double source. On comprendra facilement qu'un corps et un bras qui se développent en hauteur et en 
largeur auront une expression différente de celle d'un corps replié sur le sol. Toutefois, il serait faux de parler d'expressions définies représentées par des positions, parce que le danseur dans n'importe quelle position, peut bouger de manières très différentes. Donc, l'expression d'un mouvement dépend: de sa localisation dans l'espace, incluant la forme, et de son contenu dynamique, incluant l'effort ${ }^{59}$.

Effort n'est pas ici un terme comme un autre. C'est très exactement l'amorce d'une autre notation, le rêve d'une analytique et d'une combinatoire qui prendraient en charge le reste phénoménologique laissé par la cinétographie. Par effort, on nomme la dimension motrice du mood, une certaine attitude intérieure à l'égard du mouvement, attitude qui s'entend simultanément comme contenu de visibilité : un dedans immédiatement exposé, une qualité expressive. Cet effort, cette attitude motrice, Laban la décompose en quatre facteurs : le rapport au poids, au flux, au temps et à l'espace.

Les constituants des différentes qualités d'effort résultent d'une attitude intérieure (consciente ou inconsciente) envers les facteurs moteurs du poids, de l'espace, du temps et du flux ${ }^{60}$.

Une combinatoire qualitative peut alors se mettre en place. En instaurant des signes adéquats pour les attitudes envers les quatre facteurs, on pourra engendrer l'ensemble des attitudes globales possibles, de sorte que tout effort aura son graphe spécifique, et que tout graphe nous donnera à lire une structure d'effort ${ }^{61}$. Cette combinatoire donne lieu à quelques types élémentaires que Laban nomme actions de base. Ces actions ne désignent nullement des gestes déterminés mais encore une fois des qualités de mouvement : presser (rapport au poids ferme, rapport à l'espace direct, rapport au temps soutenu), épousseter (léger, flexible, soudain), pousser (ferme, direct, soudain), flotter (léger, flexible, soutenu), tordre (flexible, soutenu, ferme), tapoter (direct, soudain, léger), fouetter (soudain, ferme, flexible), glisser (soutenu, léger, direct) ${ }^{62}$. Nous serions donc en présence d'un alphabet des qualités, et, médiatement, d'un alphabet du sens. Cependant, cet alphabet n'est possible qu'au prix d'un abandon de l'exigence notationnelle. Soit ces actions fonctionnent réellement comme des catégories discrètes (auquel cas toute qualité de mouvement doit pouvoir être subsumée par une et une seule), soit elles ne font que poser des jalons dans la continuité infinie des dégradés qualitatifs possibles (auquel cas le principe de discontinuité graphique et sémantique au fondement de la notation devient caduque). Dans le premier cas, les graphes n'opèrent quau prix d'une violence exercée sur l'empirie. Dans le second, ils cessent purement et simplement de fonctionner comme notation ${ }^{63}$.

Cet échec dans l'exportation de la combinatoire au qualitatif et à l'expressif est la conséquence d'une impossibilité plus profonde. L'application du modèle de l'écriture alphabétique à la notation ne peut tenir ses promesses. L'articulation miraculeuse de l'analyse et du sens, de l'alphabet et de la parole, ne vaut que pour la langue et pour elle seule, et ce en vertu de la double articulation qui la caractérise. 


\section{Frédéric Pouillaude}

La notation de l'effort entend reconquérir l'expressivité et le sens, s'annexer le domaine au nom duquel l'inscription graphique s'était précisément vue condamnée. Mais, ce sens, elle se le donne dès le départ, contenu à l'avance dans les éléments mêmes de sa combinatoire. Le sens ne peut pas être engendré par les éléments, et c'est précisément pourquoi la seconde combinatoire n'est pas plus signifiante que la première; elle differe simplement par l'objet - qualitatif et expressif - qu'elle se donne, objet qui la contraint en outre à fonctionner comme une classification continue et non comme une notation. La prégnance du modèle alphabétique au sein du discours notationnel n'est que le symptôme de la dépendance proprement chorégraphique du sens à la présence; par lui, se trouve simultanément rêvée et dérobée la possibilité d'une autonomisation du sens, d'un sens absenté, par lequel l'articulation du danser à la présence se verrait enfin dénouée. Rêve qui ne peut au bout du compte qu'attester de sa propre impossibilité.

\section{Université Lille III}

\section{NOTES}

1. Pour une analyse comparative et historique des différents systèmes de notation chorégraphique, voir Ann Hutchinson-Guest, Choreo-graphics. A Comparison of Dance Notation Systems, New York, Gordon \& Breach, 1989.

2. "Notateur " est le nom moderne de ce qui s'appelait jadis, et plus proprement, "chorégraphe ». "Chorégraphe ", au $\mathrm{XVIII}^{\mathrm{e}}$ siècle, ne désigne nullement l'auteur d'un ballet, mais celui qui est capable d'inscrire graphiquement les danses. L'auteur du ballet, ou plus exactement l'auteur de ce qui dans un ballet relève de la danse, se nomme alors "maître de ballet ». Les deux fonctions ne s'excluent ni ne s'impliquent. A partir du moment où «chorégraphe » est venu se substituer à "maître de ballet", perdant ainsi sa signification purement graphique, il fallait bien inventer un nouveau terme capable de prendre en charge et d'isoler la dite signification. D'où le terme de «notateur ». Sur l'usage des termes "chorégraphe " et "maître de ballet " au XVIII ${ }^{\mathrm{e}}$ siècle, voir Jean-Georges Noverre, Lettres sur la danse et les ballets, Stuttgart, Lyon, Delaroche, 1760, et notamment la lettre intitulée "De la chorégraphie» (lettre XIII dans l'édition de 1760, lettre XXV dans la réédition par Fernand Divoire, Paris, Lieutier, 1952).

3. Raoul-Auger Feuillet, Chorégraphie ou l'art de décrire la dance par caractères, figures et signes démonstratifs, avec lesquels on apprend facilement de soi-même toutes sortes de dances, Paris, Brunet, 1700. Reprint, Hildesheim, New York, G. Olms, 1979.

4. Quelques améliorations ou réaménagements furent cependant proposés, sans grand retentissement. Voir, par exemple l'Abrégé de la nouvelle méthode pour écrire pour toutes sortes de danses de ville de Pierre Rameau (1725), ou encore l'article consacré par Cahusac à la "Chorégraphie " dans l'Encyclopédie de Diderot et d'Alembert, où Cahusac mentionne la proposition d'un certain Favier. Sur le système en général, ses traductions anglaises, et son intégration aux différents traités de danse de l'époque (notamment The Art of Dancing de Tomlinson, 1735), voir Jean-Noël Laurenti, "La pensée de Feuillet ", in Laurence Louppe (éd.), Danses tracées, Paris, Dis voir, 1991, et Catherine Kintzler (éd.), La Pensée de la danse à l'âge classique : écriture, lexique, et poétique, Villeneuve-d'Ascq, Université Lille III, Maison de la recherche, 1997 (notamment les articles de Francine Lancelot, "Ecrire la danse à l'âge classique ", Eugénia Roucher, "La belle danse : construction d'un vocabulaire par la pensée analytique ", et Jean-Noël Laurenti, "L'esprit de la méthode dans la danse française autour de $1700 »)$.

5. Recueil de dances composées par M. Pécour et mises sur le papier par M. Feuillet, Paris, Brunet, 1700, repris dans le fac-similé de la Chorégraphie, Olms, 1979.

6. Recueil de contredances mises en chorégraphie d'une manière si aisée que toutes personnes peuvent facilement les apprendre, par M. Feuillet, Paris, L'auteur, 1706.

7. Voir, là encore, les Lettres sur la danse (lettre XIII, édition de Stuttgart, 1760 ; lettre XXV, réédition Lieutier, 1952) où Noverre, outre des arguments visant le principe même de la notation, montre que le système Feuillet est devenu incapable de prendre en charge la complexité croissante des gestes et des mouvements.

8. Ann Hutchinson-Guest, dans le tableau chronologique qu'elle propose, op. cit., p. 183-184, ne dénombre pas moins d'une soixantaine de systèmes! 


\section{D'une graphie qui ne dit rien}

9. Rudolf von Laban est ordinairement considéré comme le "père fondateur " de la danse moderne. Il commence à élaborer son système de notation du mouvement dans les années 1920-1930 en Allemagne. Les principes de base donnent alors lieu à deux publications: Choreographie, Iena, Eugen Diederich Verlag, 1926, et Schrifttanz: Methodik, Orthographie, Erläuterungen, Vienne, Universal Edition, 1928. Le système fait l'objet de constantes réélaborations et débouche en 1956 sur les Principles of Dance and Movement Notation, Londres, MacDonald \& Evans. On trouvera un exposé définitif et exhaustif du système dans le Dictionary of Kinetography Laban, Plymouth, Macdonald \& Evans, 1979, élaboré par Albrecht Knust, élève et collaborateur de Laban, et, de façon plus accessible, dans le manuel de Ann Hutchinson-Guest, Labanotation, Norfolk, New Directions, 1961.

Sur la vie, l'œuvre et la pensée de Laban, on peut consulter Isabelle Launay, A la recherche d'une danse moderne, Paris, Chiron, 1997 ; John Hodgson et Valérie Preston-Dunlop, Introduction à l'œuvre de Rudolf Laban, Paris, Actes Sud, 1991 ; Valérie Preston-Dunlop, Rudolf Laban. An extraordinary Life, Londres, Dance Books, 1998; ou encore Vera Maletic, Space, Body, Expression. The development of Rudolf Laban's movement and dance concepts, Berlin, New York, Amsterdam, Mouton de Gruyter, 1987. Sur les liens entre Laban et l'Etat nazi de 1933 à 1936, voir Laure Guilbert, Danser avec le III Reich. Les danseurs allemands sous le nazisme, Bruxelles, Complexe, 2000.

10. «Avec mon ami Knust, j'ai écrit la partition d'une pièce rassemblant un millier de participants, et envoyé la notation dans les soixante villes dont étaient issus les participants. Comme nos partitions avaient été étudiées par les soixante groupes locaux, l'ensemble des participants fut à même de danser ensemble dès la première répétition, non seulement les grandes lignes, mais la chorégraphie dans tous ses détails, plutôt élaborés, et avec fort peu d'erreurs et d'interruptions " (Laban, Principles of Dance and Movement Notation, op. cit., p. 1. Nous traduisons).

11. En parlant d'échec, nous ne visons nullement la capacité descriptive des systèmes notationnels: la notation Feuillet comme la cinétographie Laban décrivent parfaitement leurs objets, la première se cantonnant au style de la «belle danse ", la seconde prenant en charge l'ensemble des mouvements corporels possibles. Encore une fois, nous ne visons que l'impossibilité historique, et sans doute conceptuelle, de leur réelle intégration aux pratiques.

12. "L'introduction d'un système notationnel pour la danse signifierait une révolution dans la production et l'exécution. Les danseurs ne seraient plus limités à l'étude et à l'exécution de leurs propres inventions, ou obligés de s'en remettre à l'imagination du maître de ballet qu'ils auraient sous la main. Les danseurs pourraient faire leur choix parmi les innombrables œuvres d'auteurs chorégraphes de talent avec lesquels ils n'auraient pas de lien personnel. Et ceux qui créent des œuvres qu'ils ne peuvent ou ne veulent exécuter eux-mêmes pourraient les écrire et les soumettre au jugement du monde chorégraphique. Les deux professions, celle d'auteur chorégraphique et de danseur, deviendraient probablement séparées" (Principles of Dance and Movement Notation, op. cit., p. 5-6. Nous traduisons).

13. Sur ce point, voir Laure Guilbert, Danser avec le III Reich, op. cit.

14. Sur l'attribution à Beauchamps de la paternité du système Feuillet, voir Jean-Noël Laurenti, "La pensée de Feuillet", op. cit., p. 104, et Jean-Michel Guilcher "André Lorin et l'invention de l'écriture chorégraphique ", Revue d'histoire du théâtre, $3^{\mathrm{e}}$ trimestre 1969. La paternité de Beauchamps semble si évidente que Laban, par exemple, ne se réfere jamais au système autrement qu'à travers le double nom de Beauchamps-Feuillet (voir par exemple, Principles of Dance and Movement Notation, p. I).

15. Sur ce point, voir les Lettres patentes du Roi pour l'établissement de l'Académie Royale de Danse en la ville de Paris, mars 1661, reproduites in Mark Franko, Dance as text. Ideologies of the baroque body, Cambridge, Cambridge University Press, 1993.

16. Un goodmanien acharné pourrait objecter qu’un tel principe sériel de multiplication des objets artistiques relève pleinement de l'autographique multiple, au même titre que la gravure ou la photographie. On répondra que la répétition sérielle des œuvres chorégraphiques, étant encore fondée sur un partage entre propriétés essentielles et contingentes, et non sur la reproduction mécanique des traits singuliers d'un prototype, ne peut en aucun cas relever de l'autographie. Cependant, dans la mesure où ce partage reste indétachable de l'histoire matérielle des filiations et qu'il lui est pour ainsi dire incorporé, il n'est pas non plus possible de parler d'allographie. Bref, comme nous le disions plus haut, l'œuvre chorégraphique n'est ni autographique, ni allographique.

17. "Figure, est de suivre un chemin tracé avec art " (Chorégraphie, op. cit., p. 2). "J'appelle le Chemin la ligne sur laquelle on dance. Le chemin sert à deux usages, premièrement il sert pour écrire les Pas et les Positions, et secondement pour faire observer la Figure des Dances" (ibid., p. 4).

18. "De la manière que l'on doit tenir le Livre pour déchiffrer les Dances qui seront écrites. Il faut savoir que chaque feuillet sur lequel la Dance est écrite représente la Salle où on dance, dont les quatre côtés en représentent les quatre côtés, savoir le haut du feuillet représente le haut de la salle, le bas du feuillet représente le bas de la salle, le côté droit du feuillet représente le côté droit de la salle, et le côté gauche du feuillet représente le côté gauche de la salle. [...] On doit remarquer qu'il faut toujours tenir le haut du Livre justement droit vis-à-vis le haut de la salle, et prendre soigneusement garde à mesure que l'on dance soit en tournant ou sans tourner qu'il ne remue point de situation. [...] Tous les pas qui se font sans tourner ou qui tournent un tour entier, on tiendra le Livre avec les deux mains par les deux côtés, mais quand il faudra tourner un quart de tour, un demi tour, ou trois quarts de tour, il sera nécessaire de prendre plus de précaution, attendu qu'il est difficile de tourner sans que le Livre tourne aussi, ce qu'il faut absolument éviter, car si le Livre sort de sa situation, il sera impossible de connaître les pas qui seront écrits" (Chorégraphie, op. cit., p. 33-34). Feuillet indique immédiatement après des règles assez complexes pour tourner sans que le livre bouge. Ainsi, pour tourner un quart de tour à droite, il faudra placer au préalable la main gauche sur le côté haut du livre, et la main droite sur le côté bas. Pour un demi tour, la main gauche devra tenir le côté droit, et la main droite le côté gauche, etc.

19. Sur la question des rapports entre représentation cartographique et orientation subjective, voir par exemple 


\section{Frédéric Pouillaude}

Merleau-Ponty, La Structure du comportement, Paris, PUF, 1942, p. 128 : «La difficulté d'une représentation pure de l'itinéraire est du même ordre que celle de lire une carte ou de s'orienter sur un plan. [...] Le tracé purement visuel exige que nous nous représentions l'itinéraire en vue cavalière, d'un point de vue qui n'a jamais été le nôtre quand nous le parcourions, que nous soyons capables de transcrire une mélodie cinétique en diagramme visuel, d'établir entre l'un et l'autre des rapports de correspondances réciproques et d'expression mutuelle. » Je remercie Maël Renouard pour la référence, ainsi que pour ses précieuses indications sur la géométrie des projections au XVII siècle (voir, sur ce point, Maël Renouard, "Le point de vue de Sirius et la cartographie du visible ", in J. Benoist et F. Merlini (dir.), Historicité et Spatialité, Paris, Vrin, 2001).

20. C'est Noverre lui-même qui emploie le terme de "géométral " pour caractériser la représentation de l'espace adoptée par la notation Feuillet. Voir Lettres sur la danse et les ballets (lettre XIII, édition de Stuttgart, 1760 ; lettre XXV, réédition Lieutier, 1952).

21. Sur cette danse "géométrique " de la fin du XVI et du début du XVII ${ }^{\mathrm{e}}$ siècle, encore appelée danse " horizontale ", voir Elizabeth McGowan, L'Art du ballet de cour (1581-1643), Paris, CNRS Editions, 1961, chap. II, et Mark Franko, Dance as text. Ideologies of the baroque body, op. cit., chap. I. Outre le Ballet de monsieur le duc de Vendosme déjà cité, il faudrait encore mentionner à titre de cas exemplaires le Ballet des Polonais (1573) et le Ballet comique de la Reyne (1581). Sur la disparition de cette fonction géométrique de la danse, nous nous permettons de citer Elizabeth McGowan, p. 37 : "Tant que les interprètes purent descendre de la scène pour danser dans la salle de théâtre, cette danse horizontale resta en vogue dans les ballets donnés à la Cour de France; elle disparaîtra seulement vers 1640 quand l'élévation de la scène rendra invisible aux spectateurs du parterre la géométrie des pas. »

22. Chorégraphie, op. cit., p. 103-104. Nous soulignons. Voir aussi p. 95 : «Je dis donc que lorsque dans une dance on dance dans quelque espace de temps dans une même place, on ne doit plus regarder le chemin que comme le conducteur des Pas et non de la Figure; mais quand la Dance va toujours et ne reste point en place, pour lors on doit regarder le Chemin non seulement comme le conducteur des Pas, mais encore de la Figure. " Pour ce qui est du lien entre le chemin et la mesure musicale, voir p. 87 : «Les Mesures des Dances seront marquées comme on les marque à la Musique, c'est-àdire par des petites barres coupant le Chemin en travers qui représentent les même barres qui tranchent les cinq règles de la Musique, dont les entre-deux seront autant de mesures."

23. Chorégraphie, op. cit., p. 9. Nous soulignons.

24. Nous reprenons ici deux des critères proposés par Nelson Goodman dans sa théorie de la notation, et qu'il applique lui-même localement à la notation Laban. Voir Langages de l'art, trad. fr., Paris, Chambon, 1990, chap. 4 et 5.

25. Les basses-danses furent en usage depuis le début du XV jusqu'au milieu du XVI ${ }^{\mathrm{e}}$ siècle. Elles reposent sur cinq pas de base (révérence, simple, double, branle, reprise), que l'on abrège par les lettres suivantes : $R, s, d, b, r$. On peut alors noter les danses en combinant simplement les lettres. Ce système de notation, présent dans le Manuscrit des basses-danses de Marguerite d'Autriche (1460, reproduit par l'American Library of Congress), se retrouve également dans un imprimé anonyme de la fin du XV siècle : L'Art et Instruction de bien dancer. Pour ce qui est du système Lorin, voir Jean-Michel Guilcher, "André Lorin et l'invention de l'écriture chorégraphique », loc. cit.

26. Chorégraphie, op. cit., p. 11.

27. "Pour poser les signes en leurs lieux et places, il faut auparavant reconnaître le Pas en ses trois parties, savoir son commencement, son milieu et sa fin" (Chorégraphie, op. cit., p. 13).

28. Voir notamment Jean-Noël Laurenti, «La pensée de Feuillet », loc. cit.

29. Les Mots et les Choses, Paris, Gallimard, 1966, p. 76-77.

30. Ibid., chap. III, section VI, "Mathesis" et "Taxinomia ".

31. La formule "rhétorique muette " est tirée du De Saltatione de Lucien et se retrouve dans à peu près tous les traités de danse, depuis la fin du XV siècle jusqu’à Ménestrier (Des ballets anciens et modernes selon les règles du théâtre, Paris, Guignard, 1682), et au-delà. La signification cosmique du ballet est omniprésente à la Renaissance; on la retrouve encore à l'Age classique, par exemple, chez Mersenne. Voir Harmonie universelle, 1636, Traité des chants, Proposition XXII : «Je dis premièrement que l'on peut faire des ballets qui représenteront et enseigneront l'Astronomie. " Cette fonction représentative et didactique du ballet permet par ailleurs de considérer la danse comme une réelle langue universelle : «A quoi j'ajoute que [les danses] par lesquelles on représentera les sciences et les Arts libéraux, seront aussi bien reçues et entendues des Chinois et de toutes les autres nations que les Français, et conséquemment elles pourront servir d'une langue commune, naturelle et universelle, par le moyen de laquelle le commerce, les intelligences mutuelles et l'amitié réciproque pourront être établies et conservées dans le monde» (p. 160. Nous soulignons).

32. Louis de Cahusac, Traité historique de la danse, La Haye, Neaulme, 1754, Livre IV. Sur le concept de "danse en action " chez Cahusac, voir Catherine Kintzler, "La danse, modèle d'intelligibilité dans l'opéra français de l'âge classique", in La Pensée de la danse à l'âge classique : écriture, lexique, poétique, op. cit.

33. Noverre, Lettres sur la danse et les ballets (lettre X, édition de Stuttgart, 1760 ; lettre XXII, réédition Lieutier, 1952, p. 187). Nous citerons désormais les Lettres dans la numérotation et la pagination de la réédition Divoire, Lieutier, 1952.

34. Ibid., lettre I, "Renaissance de l'art de la danse ", p. 12. Nous soulignons.

35. Ibid., lettre V, "Division de la danse ", p. 37. Hormis le premier paragraphe et "danse en action" au troisième, nous soulignons.

36. "J'appris à la danse muette à articuler; à exprimer les passions et les affections de l'âme. » Ibid., lettre I.

37. Ibid., lettre XVI, «Défaut de nos premiers ballets", p. 113. Nous soulignons.

38. Claude-François Ménestrier, Des ballets anciens et modernes selon les règles du théâtre, op. cit.

39. Cahusac, Traité historique de la danse, op. cit., seconde partie, Livre III, chap. IV, "Vices du grand ballet », p. 47 - 


\section{D'une graphie qui ne dit rien}

49. Ce caractère pictural et instantané de la signification chorégraphique est déjà attesté par Ménestrier en 1682 : «C'est que le même personnage ne doit paraître qu'une seule fois, du moins sous le même habit. La raison de cela est que le Ballet ne représentant que par les figures et les mouvements, quand le personnage paraît une seconde fois, il n'exprime rien de nouveau quant à la figure, et il faut que les mouvements soient diversifiés, que l'on puisse entendre ce qu'il représente de nouveau. " (Des ballets anciens et modernes, op. cit., p. 141-142.)

40. Lettres sur la danse et les ballets, op. cit., XIII, p. 97-98. Hormis le premier paragraphe et le vers, nous soulignons.

41. «Le personnage le plus recommandable de la Chine est celui qui sait une plus grande quantité de mots. L'érudition de ce Pays n'effleure pas même les choses. Un lettré passe sa vie à mettre, à arranger dans sa tête un nombre immense de paroles isolées, et les savants de la Chine déclarent qu'il est savant. Je crois voir un homme qui ayant dans sa main la clef du Temple des Muses, consume ses jours et toute son adresse à la tourner et à la retourner sans cesse dans sa serrure, sans oser jamais toucher au ressort. Tel est notre meilleur danseur " (Traité historique de la danse, op. cit., seconde partie, livre IV, chap. 4, p. 129). "Un Maître Ecrivain est un expert qui enseigne à faire des lettres. Un maître à danser est un artiste qui montre à faire des pas. Le premier n'est pas plus éloigné de ce que nous appelons dans la Littérature, un Ecrivain, que le second l'est de ce qui peut mériter au théâtre le nom de danseur » (ibid., chap. 8, p. 144).

42. Lettres sur la danse et les ballets, op. cit., XXII, p. 192-195.

43. Ibid., II, p. 20.

44. "Que mes confrères se persuadent que j'entends par gestes les mouvements expressifs des bras soutenus par les caractères frappants et variés de la physionomie", ibid., XXII, p. 190.

45. Ibid., XXII, p. 188-189.

46. Ibid., XXV, « De la chorégraphie».

47. Ibid., XXV, p. 224. Hormis "Chorégraphie", nous soulignons.

48. Ibid. Nous soulignons.

49. Ibid.

50. Ibid., XXV, p. 225. Nous soulignons.

51. Ibid., XXV, p. 231-232. Hormis "chorégraphiquement", nous soulignons.

52. Ibid., XXV, p. 230. Hormis "chorégraphie" et "costume ", nous soulignons.

53. Voir Principles of Dance and Movement Notation, 1956, op. cit., p. IX et XI; Modern Educational Dance, Londres, MacDonald \& Evans, 1948, trad. fr., La Danse moderne éducative, Bruxelles, Complexe-CND, 2003, p. 19.

54. Principles of Dance and Movement Notation, p. 4 . Nous traduisons et soulignons.

55. La Danse moderne éducative, op. cit., p. 44. Nous soulignons.

56. Ibid., p. 63. Nous soulignons.

57. Pour un exposé détaillé de la cinétographie, voir par exemple Jacqueline Challet-Haas, Grammaire de la notation Laban, 2 vol., Paris, CND, 1999.

58. Modern educational dance, op. cit., p. 44, trad. fr. p. 63. Nous soulignons. La traduction proposée par Jacqueline Challet-Haas et Jean Challet a pris le parti de traduire le difficile mood tantôt par « expression ", tantôt par "couleur ". 59. Modern educational dance, op. cit., p. 45-46, trad. fr., p. 64. Nous soulignons.

60. The Mastery of Movement, 1950, trad. fr., La Maîtrise du mouvement, Paris, Actes Sud, 1988, p. 34. Nous soulignons.

61. Sur les graphes d' "effort", voir Effort, en collaboration avec F. C. Lawrence, Londres, MacDonald \& Evans, 1948; La Danse moderne éducative, op. cit., p. 74-104; La Maîtrise du mouvement, op. cit., p. 105-118.

62. Les actions de base résultent de la combinaison de trois facteurs seulement: poids, temps, espace. Le flux s'y adjoint de manière accessoire. Voir La Danse moderne éducative, op. cit., p. 75-77.

63. Sur la discontinuité sémantique et graphique comme critère de la notation, nous nous permettons de renvoyer encore une fois aux analyses de Nelson Goodman dans Langages de l'art, op. cit., chap. 4 et 5. 